\title{
Un cinéma en travail ? À partir du film de Jean Rouch et d'Edgar Morin Chronique d'un été
}

A cinema at work? Based on Jean Rouch and Edgar Morin's film Chronique d'un été

Marc-Henri Piault

\section{OpenEdition}

\section{Journals}

Édition électronique

URL : https://journals.openedition.org/itti/1659

DOI : 10.4000/itti. 1659

Éditeur

Université de Poitiers

Référence électronique

Marc-Henri Piault, « Un cinéma en travail ? À partir du film de Jean Rouch et d'Edgar Morin Chronique d'un été », Images du travail, travail des images [En ligne], 11 | 2021, mis en ligne le 01 septembre 2021, consulté le 19 février 2022. URL : http://journals.openedition.org/itti/1659 ; DOI : https://doi.org/ 10.4000/itti.1659

Ce document a été généré automatiquement le 19 février 2022.

Images du travail, travail des images 


\title{
Un cinéma en travail ? À partir du film de Jean Rouch et d'Edgar Morin Chronique d'un été
}

\author{
A cinema at work? Based on Jean Rouch and Edgar Morin's film Chronique \\ d'un été
}

Marc-Henri Piault

\section{Avant-propos}

1 En hommage à Marc-Henri Piault, décédé le 4 novembre 2020, le Comité de rédaction de la revue Images du Travail, Travail des images a décidé, avec l'aimable autorisation des éditeurs, de publier sa contribution à l'ouvrage collectif éponyme: «Un cinéma en travail ? À partir du film de Jean Rouch et d'Edgar Morin Chronique d'un été » in Géhin J.P. \& Stevens H. (dir.) coédité en 2012 par les éditions Atlantique à Poitiers et les Presses Universitaires de Rennes.

Depuis son origine, Marc-Henri Piault a participé au projet Images du Travail, Travail des Images. Il a contribué au colloque international en novembre 2009 à Poitiers et fut un collaborateur important de la revue dont il était membre du conseil scientifique. Plus largement, observateur attentif des interactions entre cinéma et anthropologie, il laisse une publication de référence "Anthropologie et cinéma: passage à l'image, passage par l'image " qui est aujourd'hui encore un repère incontournable pour comprendre les évolutions que suscite la rencontre entre le cinéma et l'anthropologie au sein de la recherche en sciences humaines et sociales (SHS) tout au long du $\mathrm{XX}^{\mathrm{e}}$ siècle. 
Image 1 : Portrait de Marc-Henri Piault

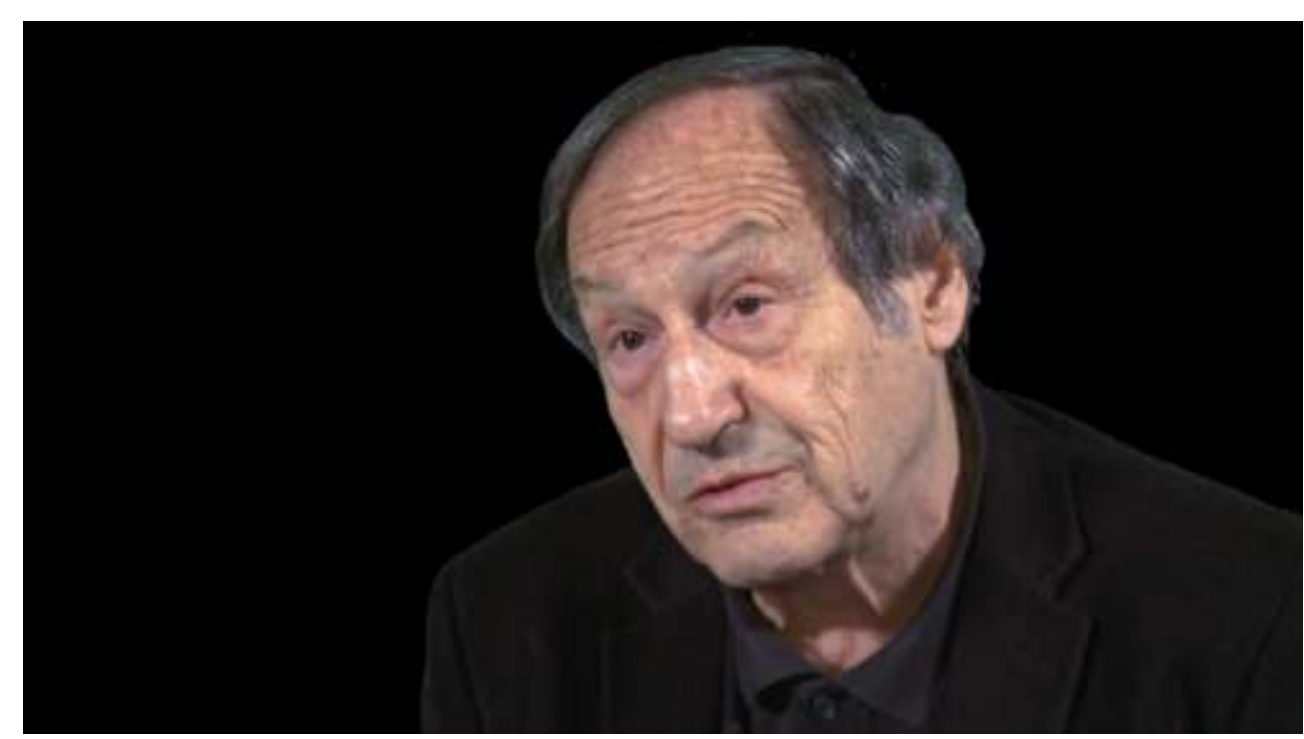

(c) Pascal Cesaro

3 Anthropologue, cinéaste et directeur de recherche au CNRS, ses terrains l'ont mené à travers le monde, de l'Afrique occidentale (migrations, formations politiques précoloniales, cultes de possession) à la France (identité régionale et représentation culturelle) et au Brésil (croyances, appartenances, identités et conflits). Il a présidé le Comité du film ethnographique au sein duquel il transforme le nom du festival en l'honneur de son créateur Jean Rouch et afin de l'ouvrir plus largement aux questions de cinéma. Il fut responsable de l'Association Française des Anthropologues, du Journal des Africanistes, du Journal des Anthropologues et l'auteur d'une centaine de publications en français, anglais, portugais, espagnol et italien.

4 La contribution de Marc Henri Piault repose sur une conférence qu'il a réalisée lors de la soirée inaugurale de la première édition du festival Filmer le travail durant laquelle a été projeté Chronique d'un été. Cette conférence grand public est l'occasion de rappeler brièvement l'histoire de ce projet de médiation scientifique des sciences humaines et sociales visant à mobiliser le cinéma et plus largement les pratiques artistiques comme la photographie, la bande dessinée, la littérature, le théâtre, la musique... pour dynamiser la réflexion et le débat sur l'évolution du travail contemporain. Cette volonté d'échanges et de croisement des points de vue artistiques et scientifiques a débouché sur l'organisation à Poitiers chaque année depuis 2009 du festival de plus en plus reconnu, Filmer le travail. L'aspect le plus scientifique de ce projet a été l'objet de deux colloques internationaux Images du Travail, Travail des Images en 2009 et 2013 et de l'ouvrage éponyme publié en 2012. Depuis 2016 la revue scientifique, semestrielle, pluridisciplinaire, numérique et ouverte Images du Travail, Travail des Images poursuit l'aventure en étant le lieu où les recherches, les analyses et les méthodologies mobilisant des images du travail sont exposées et débattues. 


\section{Un cinéma en travail ? À partir du film de Jean Rouch et d'Edgar Morin Chronique d'un été}

5 Un important critique de cinéma français écrivait en 1961 que le film Chronique d'un été montrait notamment "le dégoût d'un univers professionnel devenu inhumain et la

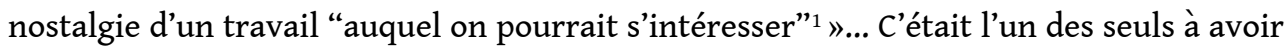
perçu dans ce film ce qui, à le revoir bien plus tard, en fait une œuvre prémonitoire sur ce plan. C'est en effet l'une des premières expressions d'un malaise qui commence à saisir la jeunesse française mais aussi la classe ouvrière à une époque où la réflexion politique prédominante restait marquée par la guerre froide sur le plan international et les guerres coloniales sur le plan strictement français.

6 La France est en train de subir une transformation radicale. La majorité des paysans a quitté la terre pour les usines récemment reconstruites et peuple désormais les villes se relevant lentement des ruines de la libération. Automobiles et transports routiers dessinent une nouvelle géographie de la circulation et des échanges, l'agriculture s'industrialise et les macro entreprises de distribution commerciale se développent. Le monde extérieur à nouveau s'ouvre et les distances séparant pays et continents se rétrécissent à mesure de l'accélération des communications. Les différents modes d'existence et de comportement se diffusent et s'identifient à travers une jeunesse tournant le dos à la guerre et à ses rigueurs qu'elle a peu ou pas éprouvées. Elle découvre le désir, le plaisir, le voyage et les premières tentations du consumérisme mais aussi les premiers doutes relatifs au " progrès » unidirectionnel d'une civilisation et à l'unicité rationnelle du savoir.

7 Le modèle au quotidien devient américain. Au-delà des partis pris politiques séparant encore le monde entre gauche et droite, bons et mauvais (suivant les points de vue!), les États-Unis offrent les voies d'une nouvelle culture laboratoire et libératoire par rapport aux carcans des sociétés petites-bourgeoises européennes encore marquées par les systèmes de mesure d'avant la guerre mais traumatisées par la violence d'une guerre dont elles n'avaient pas su, pas pu ou pas voulu reconnaître l'inhumaine radicalité. Le cinéma, la peinture, la musique - le jazz et le rock en particulier -, la littérature, l'architecture, tout semble venir d'Amérique ${ }^{2}$. Ainsi se complètent, dans une opposition qu'on espérait dialectique, les images américaines du modernisme et de la révolution technologique en cours avec ce qui parait le mouvement avant-coureur de nouveaux bouleversements politiques, sociaux et culturels manifestés par la récente diffusion du maoïsme et l'impact fulgurant du castrisme. Simultanément se font entendre les premières sonorités discordantes des langages et des comportements apparemment erratiques de la beat generation. Il y avait alors, masqué par la division violente et imposée du monde en deux blocs antagonistes, le désir mal exprimé d'une mondialisation qui aurait été celle des êtres humains et du partage de leurs façons d'être, de leurs cultures, de leurs valeurs, avant de devenir ce qui est aujourd'hui proposé sinon imposé, l'amalgame misérable des manières de produire, d'acheter, de vendre et de consommer sous le contrôle des banques et des consortiums internationaux.

8 Dans ce contexte, la naissance du cinéma direct dans les années soixante paraît un moment privilégié d'une prise de conscience : il y aurait la possibilité de rencontrer l'autre sans l'assimiler ou le dominer. Notre curiosité aurait alors le statut d'un désir de correspondance et non plus de dévoration. Sans doute s'agissait-il encore d'une 
attitude naïve assumant l'évidence d'un désir partagé malgré les distances économiques, politiques et sociales et sans tenir compte de la vivacité des préjugés identitaires et d'appartenance. Comme le disait Jean Rouch $(1962,48)$ à propos de Jean Vigo, il s'agissait « d'utiliser la caméra libre pour montrer simplement les gestes de ses contemporains, et... leur culture »... C'était l'émergence d'une attitude qui n'était plus celle de la découverte d'une altérité exotique et plus ou moins archaïque mais celle d'un entendement de l'autre dans sa différence aussi bien que sa proximité.

\section{L'entreprise d'un film : le cinéma c'est la vie}

Anticipant sur des développements technologiques en cours, Jean Rouch déclarait que les rêves de Dziga Vertov et de Robert Flaherty se combineraient dans une caméra participante «œil-oreille» qui passerait naturellement entre les mains de ceux qui jusqu'alors s'étaient toujours trouvés devant son objectif. Ainsi, disait-il, l'anthropologue ne monopoliserait plus l'observation et, à son tour, lui et sa culture seraient l'objet du regard de l'autre (Rouch, France, 1979). Ces réciprocités sont peu à peu entrées dans les mœurs. L'irréductibilité de l'autre n'est plus une ultime résistance passéiste à l'ordre triomphant de la modernité en marche, du progrès ou, plus récemment, du développement en cours. Il énonce une revendication alternative, une proposition qui, sans être plus ou moins "vraie", se révèle simplement légitime en exprimant le point de vue spécifique d'une formation sociale donnée. Cette résistance, comme la lutte anticoloniale, relativise la raison dominante qui n'est plus que la raison du dominant et donc susceptible d'être questionnée sinon combattue tout autant que la dominance elle-même.

L'expérience menée par Jean Rouch et Edgar Morin, avec Chronique d'un été, n'est pas un renversement des rapports entre les sociétés mais elle soumet la société des réalisateurs, celle qui jusqu'alors interrogeait les autres par rapport à sa propre norme, à une interrogation sur elle-même (Piault, 2004). C'est un bouleversement des rôles habituels du cinéma d'enquête: acteurs et réalisateurs du film se mettent en jeu, questionnent leurs intentions de vie mais aussi de production cinématographique. Le déroulement du film devient la question principale. La tension narrative ne vient pas d'une condition extérieure ou d'une mise en scène thématisée, elle surgit des relations établies entre les différents protagonistes au cours du tournage.

Ce média ne peut être affiché ici. Veuillez vous reporter à l'édition en ligne http:// journals.openedition.org/itti/1659

Une sorte d'enquête d'opinion, menée dans les rues de Paris, sert de prétexte à l'entrée en réalisation du film au cours duquel des personnages peu à peu s'exposent. Les différents caractères vont se rencontrer, s'affronter ou s'accorder, d'abord à travers les images des uns vus par les autres lors d'entrevues qui ouvraient les premières conversations, puis au cours de longs dîners d'interrogations réciproques mêlant l'évocation des destins individuels à la finalité et à la construction du film. Attitudes et comportements se composent à l'image par les acteurs comme par les réalisateurs, jouant leur propre rôle. Les méthodes de travail, la place de la caméra, son effet sur la sincérité ou le naturel des protagonistes parfois directement mis en cause, font partie de la trame du film qui se construit de sa propre construction. Il y a glissement de la 
réflexion à l'action, de la mise en place d'une situation, d'un climat psychologique à la performance elle-même. Le film se nourrit de son déroulement, il se compose et s'offre comme véritable travail imagétique.

Ainsi en est-il de la réflexion solitaire de l'un des personnages, Marceline. À Paris, vidé de ses habitants par les vacances du mois d'août, la jeune femme avance seule au milieu de la Place de la Concorde déserte puis dans un pavillon vide des Halles. S'éloignant de la caméra, elle enregistre un récit à l'aide d'un magnétophone portable, synchrone avec l'image. À la fin de la séquence, les réalisateurs constatent qu'elle a fait une évocation dramatique de son retour du camp de concentration où, petite fille, elle avait été enfermée pendant la guerre. Jean Rouch, bouleversé comme la plupart des participants au film, évoquera à ce sujet l'idée d'un "sacrilège spontané ", l'émergence imprévue dans des conditions et à l'intérieur d'un cadre provocant, d'une mémoire irrépressible, aux effets incontrôlables et peut-être dangereux. On aurait ainsi, par l'effet d'une mise en scène hyper suggestive et contraire à toute spontanéité ${ }^{3}$, le surgissement paradoxal de l'inattendu, du direct le plus profond, de l'affect, du sentiment, de l'émotion. À l'inverse, Marceline assure qu'elle a préparé son texte, influencée par le film alors récent d'Alain Resnais, Hiroshima mon amour (1959). Toute la séquence aurait été de sa part un jeu sans implication affective directe mais destiné à réussir une performance cinématographique ${ }^{4}$. Elle se trouvait en effet, au cours du tournage, en compétition évidente avec une autre jeune femme, manifestement très sensible et qui avait, avant elle, enregistré une séquence particulièrement touchante sur sa vie sentimentale. Il y avait un défi à relever pour emporter a posteriori le rôle principal dans un film en principe sans acteur. On voit donc ici le jeu actif des protagonistes d'un film, la multiplicité des actions en cours et des motivations à l'œuvre pour exprimer tel ou tel type de sentiment, pour manifester tel ou tel type d'attitude. En ce sens, le film est bien réalité et travail imagétique: il provoque des situations spécifiques, sa réalisation même engendre des rapports concrets entre les personnes concernées. Leurs effets sont nécessairement sensibles dans le traitement narratif et l'orientation diégétique elle-même.

\section{Le travail du temps}

14 Chronique d'un été n'est pas seulement l'avènement du cinéma direct en France et la marque d'une aventure poursuivie également aux États-Unis, au Canada ${ }^{5}$, en Australie et en Afrique. C'est un film-action où se nouent des relations effectives entre des protagonistes réunis de manière plus ou moins artificielle. L'intelligence de Rouch et de Morin est d'avoir conduit le spectateur au travers des implications réciproques des acteurs et des réalisateurs. Ils proposaient une sorte d'anthropologie dynamique d'une société émergente. Le réalisateur n'est plus démiurge ou savant montreur d'ombres mais médiateur impliqué par les effets de son entreprise. C'est une des choses que, le temps ayant passé, nous pouvons mieux apprécier : la capacité à laisser se développer les contradictions et les oppositions dans le cours vécu du tournage.

La réalisation de Chronique d'un été est une double leçon de l'anthropologie rouchienne : proximité et continuité donnent à voir et conduisent à percevoir le sens de la différence, échanger les points de vue donc "changer et décentrer l'analyse ». L'anthropologie partagée met en perspective l'anthropologue: sa démarche s'inclut dans le questionnement. Enquêteur et enquêté sont englobés dans une situation qui 
leur échappe à mesure qu'ils la définissent. Sans doute est-ce la raison pour laquelle Morin, désemparé par la réalisation chaotique d'un film qui ne suit aucune des intentions des auteurs et par l'ambiguïté des réactions qu'il va susciter, s'étonne sans parvenir à se rassurer tout à fait sur la nature de ce qu'il persiste à appeler « cinéma vérité » : « ce film est hybride ", écrit-il à plusieurs reprises dans un article relatant son expérience $(1962,35,37)$. Il sent que les catégories alors régnantes ne conviennent plus tout à fait et que le film est l'expression de cette remise en cause émergente. "Film bourgeois ou révolutionnaire? » Posant cette question, il réalise qu'elle n'est pas pertinente car « le sens du film est clair si l'on conçoit qu'il conteste en même temps les valeurs régnantes de la société bourgeoise et les stéréotypes staliniens ou pseudoprogressistes» $(1962,39)$.

16 Cette perplexité empêche sans doute Morin d'approfondir les questions émergeant au cours du film. Il en fait l'inventaire mais reste débordé par la situation elle-même, le sociodrame en cours. La réalité du tournage lui paraît empêcher l'épuration des questions significatives qu'en bon sociologue il aurait voulu voir approfondir sinon résoudre. Il passe en effet des intentions de réalisation et des débats sur le montage, c'est-à-dire sur l'importance à accorder aux êtres et aux évènements, à l'orientation significative d'un film échappant aux représentations préalables de ses auteurs. S'agit-il d'un malentendu initial entre Rouch et Morin ? Ce dernier désire montrer et démontrer quelque chose et pour Rouch, un film est le fruit d'une expérience de terrain et d'une intuition qui doit se développer en cours de réalisation: "Le film est pour moi un moyen d'expression total et je ne vois pas la nécessité... d'écrire avant, pendant ou après le tournage » $(1962,52)$. On voit bien, dans leur différence de points de vue, un élément moteur $\mathrm{du}$ film, ce qui lui donne une tension faisant apparaitre des interrogations fondamentales et des positions sinon des oppositions caractérisées. Rouch questionne, provoque tandis que Morin interprète et suggère. Morin attend que les personnes s'expliquent, se dévoilent et éventuellement se regardent réciproquement, Rouch attend quelque chose de leurs rencontres.

Entre ces deux positions, les acteurs se défendent ou se mettent en scène, chacun répondant par une référence spécifique à la demande des réalisateurs. L'une des deux héroönes, Marilou, est sensible, émotive ; l'autre, Marceline, est calculatrice, cérébrale. Toutes les deux sont tendues et offrent à la caméra une part de leur émotion profonde, directement exprimée par Marilou, savamment mise en scène par Marceline. Le débat reste ouvert pour apprécier l'immédiateté et le degré de profilmie de l'une et de l'autre. Cinquante années plus tard, nous ne cherchons plus à savoir où est la vérité ni même s'il y aurait des moyens privilégiés pour aborder cette vérité. Nous avons accepté la mise en scène comme partie intégrante de notre propre existence. Par contre, nous découvrons encore en ce film ce qui gênait certainement tout autant Morin que Rouch : l'ambiguïté permanente des gestes et des paroles. L'hétérogénéité dont Morin se plaint n'est pas celle du film, elle est celle des personnes, intrinsèque à la réalité multiple de chacun d'entre nous. Nous commençons seulement à la reconnaître sinon à l'accepter.

\section{Le travail et la vie}

18 Cette hétérogénéité des constituants du film qui trouble tellement Morin en est certainement l'un des aspects essentiels, ce qui lui donne à la fois sa résonance historique et sa permanence comme questionnement existentiel. Aucun des acteurs 
n'offre une théorie de l'existence, une instrumentation décisive du savoir. Tous au contraire mettent en question la plupart des certitudes qui les ont entourés, qui ont contribué à leur formation. Tous échappent ou cherchent à échapper à ce qu'à l'époque on aurait appelé leur condition sinon leur conditionnement. Dans tous les domaines le doute est à l'ordre du jour. Morin le suggère, la vraie question « est de savoir s'ils (les acteurs du film) posent ou non des problèmes profonds et généraux, qui sont ceux du travail aliéné, de la difficulté de vivre, de la solitude, de la recherche d'une foi » $(1962,38)$.

Ce sont les questions essentielles qui vont s'exprimer de plus en plus fortement dans les décades suivant la réalisation du film, non seulement en France mais dans une grande partie des pays du monde. Le film en cours de réalisation ne proposait pas aux acteurs ni aux réalisateurs une sorte de bilan de leurs existences mais bien au contraire une conjecture d'avenir. C'est à cela qu'ils ont répondu, mettant en question ce qui dans leur temps présent paraissait souvent un empêchement et, plus rarement, une incitation à la vie. Et singulièrement, en ces temps initiaux de la sécurisation économique occidentale, la mise en question la plus longuement traitée par les acteurs du film, portait sur le travail dont j'ai relevé la qualification à travers les différentes séquences qui en traitent explicitement ${ }^{6}$ :

- Marceline : je travaille... Je fais des enquêtes psychosociologiques...

Rouch : ça vous intéresse.

Marceline: pas du tout (séquence $1: 56$ ).

- La femme du garagiste : pour arriver dans la vie à quelque chose, pour faire quelque chose soimême, il faut travailler (séquence $3: 67$ ).

- Le garagiste: ils sont fous les gens... Ils travaillent toute la semaine... Ils font plus rien le dimanche... (nous) on s'intéresse à des choses inutiles... Uniquement pour s'amuser (séquence $3: 68$ ).

- Maddie (artiste) : j'ai décidé avec des amis de monter une affaire qui devait nous faire faire fortune rapidement... Parce que travailler beaucoup, ce n'est pas très intéressant... (séquence $4:$ :70-72).

- Jacques : j'ai jamais entendu un mec qui me dise que son boulot l'intéressait... le travail est tellement parcellé... monotone... emmerdant... toujours le même.

- Angelo : moi je fais $24 \mathrm{~h}$ par jour. Parce qu'on fait 9 heures, par jour, c'est vrai, mais le restant des heures on s'en sert pour dormir, or on dort pour aller travailler... (séquence $5: 74-76$, les ouvriers).

- Landry (étudiant africain) : ... il me viendrait jamais à l'idée de travailler dans une usine... dans une usine vous êtes là... enfermés... toute la journée... (séquence 7 : 79).

- Angelo (ouvrier) : En France... le type... travaille pour lui... il se prive de certaines choses... il veut jouer... le mec s'est privé pour avoir un costume... c'est de la rigolade parce que le lundi il va recommencer comme un pauvre type dans une usine crado, craspèque... (séquence 7 : 81).

- Gabillon (employé) : Pour moi le travail c'est du temps perdu (séquence : 85)... le drame de notre époque c'est qu'on choisit de moins en moins son travail... on n'entre pas dans quelque chose, on tombe dans quelque chose... un travail qui n'intéresse pas... dans lequel on ne trouve aucun intérêt... qui n'a aucun sens... il faut le subir... jusqu'à six heures du soir... et puis après on est un tout autre homme, une tout autre personne... il faut diminuer cette participation... au travail et donner toujours davantage à côté, ce que j'appelle la vie marginale... (séquence $8: 86-87$ ). 
- Les ouvriers (élément de la discussion coupé dans le montage final) : moineau : ... ce garçon... aime son travail et tu peux l'amener n'importe où et lui donner deux briques par an, il n'ira pas (136).

- Jacques: la question ce n'est pas une question simplement d'argent, c'est une question de travail... Trouver un boulot qui satisfasse, un boulot dans lequel enfin les gens se réalisent... (137) ... le fait de sortir c'est formidable... enfin, il y a le type qui dit moi je suis venu ici pour m'en aller, ben c'est peut-être vrai, c'est foutre le camp de là enfin... quand on part et qu'on laisse des types derrière, c'est vraiment un sentiment de bien-être (139).

Jean : c'est montrer l'imbécillité du travail... j'ai l'impression d'être à l'armée... (139).

Jacques: À partir d'un certain âge... toute leur vie est orientée vers ce but... arriver à la retraite... l'issue... c'est de me révolter... quand on arrive à faire une grève... C'est arrivé rarement mais c'est arrivé... les chefs... ils se demandaient ce qui arrivait, et enfin ils ne savaient plus, c'était nous... (142-143).

Jean : La grève pour les jeunes c'est ridicule... je m'en fous complètement (143)... dans le civil on a l'impression d'être libre alors qu'on n'est pas libre du tout (144).

On voit bien ici une image débilitante du travail, contrainte insurmontable et aliénante sauf dans le cas évoqué par Moineau d'un jeune homme refusant un travail mieux payé pour rester indépendant. Cela confirme l'appréciation portée sur une survie quotidienne assurée par un travail imposé et en rupture avec ce qui serait une «vraie vie ». Il y aurait opposition sinon contradiction radicale entre vie et travail. L'image la plus forte en est donnée par Jacques évoquant un ouvrier qui ne supporte de travailler qu'à cause du moment où il quitte l'atelier en laissant derrière lui ses collègues.

Cette description paradoxale paraît exprimer une haine du soi aliéné, une coupure profonde et pratiquement irrémédiable de la personne.

\section{Le travail au cinéma}

Cette rupture semble rejoindre, jusque dans l'image proposée, un point de vue exprimé au journal Le Monde (5 mars 2003) par le ciné-documentariste Jean-Louis Comolli voulant démontrer qu'au cinéma les réalités du travail étaient absentes sinon simplement dénoncées. Il utilisait pour cela l'un des documents symboliques de la naissance du cinéma tourné et présenté en 1894, La Sortie des usines Lumière. On y verrait, selon lui, des ouvrières «tourner le dos au travail». Le travail appartiendrait donc à l'origine même du cinéma et lié à un débat qui ne cessera de se complexifier sur une relation qui serait conflictuelle entre l'art et l'industrie.

En fait, Comolli opère un raccourci sémantique, identifiant un mouvement des corps (les ouvrières sortent de l'usine et, face à la caméra, tournent donc évidemment le dos à la façade des établissements Lumière symbolisant le travail) avec une action supposée délibérée d'ouvrières récusant (l'expression tourner le dos signifie bien en français une position consciente de refus) leur travail. Cela reviendrait à dire que chaque fois que je ferme les yeux, j'exprime un refus ou un désir de fuite de la réalité dans son ensemble! C'est également une négation de ce qu'est l'image animée dont, depuis Dziga Vertov et Lev Koulechov, on sait qu'elle prend sens dans une relation établie par le réalisateur avec d'autres images et interprétée par le spectateur. Enfin il y a un certain coup de force visant, à partir d'une interprétation très particulière et avec des exemples peu représentatifs, à établir une relation définitive entre ce qui serait l'essence du cinéma et un concept social central comme le travail. 

Wonder, tourné à une époque symbolique, 1968 : après une longue grève, les ouvriers décident d'assumer la gestion d'une entreprise abandonnée par ses patrons. Une ouvrière y manifeste vivement son dégoût pour le travail, signifiant clairement l'un des aspects fondamentaux de cette crise de 1968 durant laquelle se sont exprimées largement des positions jusqu'alors sporadiques et souvent réprimées autant à droite qu'à gauche : elles mettaient en cause la valeur intrinsèque du travail et surtout le travail comme valeur. C'était, rappelons-le, l'apparition des premiers indices publics d'une crise idéologique radicale entrant dans le complexe des raisons qui ont contribué à la chute de la plupart des régimes communistes et à la formulation d'une nouvelle pensée dominante, celle de la globalisation.

il serait facile de montrer la présence relativement fréquente du travail dans les films, tant de fiction que documentaires, autant pour en montrer la grandeur que pour en dénoncer les aliénations et les horreurs.

Il serait aussi bien long de faire un inventaire des réalisations montrant les conditions concrètes de la production ainsi que l'univers social, ce «monde du travail» dont la présence marque, par exemple, une bonne partie de ce que l'on a appelé le « réalisme poétique " français des années trente, identifie le "cinéma social » britannique à la même époque (Piault, 2000) ou bien entendu la production soviétique et les grands films sociaux nord-américains des années 1940-1950. Vertov avec L'Homme à la caméra (1929) et Symphonie du Donbass (1930) ; Ruttmann dans Berlin, symphonie d'une grande ville (1927) ; Chaplin avec Les temps modernes (1936) Lang et Metropolis (1927); Vigo réalisant L'Atalante (1934) ou bien encore, Grierson souhaitant «porter la classe ouvrière à l'écran... »

Pas plus que de traiter du cinéma en général, il n'est intéressant de faire la liste des films qui traiteraient du travail ouvrier en général dans la perspective illusoire de donner un sens à ce que serait la relation cinéma-travail dont une identification reste peu probable. Cependant, la façon de traiter certains aspects des relations des personnes avec les modalités de leur subsistance renvoie aux différentes acceptions possibles de cette relation et c'est également sinon surtout une question politique relative aux critères de son identification et à sa maîtrise. Le travail, comme activité singulière, distinctive, induit nécessairement une réflexion sur les formes d'articulation sociale qui le permettent et qui en sont le produit aussi bien que sur les instances qui le gèrent. Il y a donc un engagement heuristique à s'interroger sur les modes de sa représentation et en particulier dans le cinéma. C'est un lieu stratégique pour une explicitation des théories sociales à l'œuvre ici ou là dans les pratiques vécues par les différents partenaires en cause. C'est également l'occasion d'une interrogation sur les rôles possibles du cinéma, c'est-à-dire des cinéastes autant que de la chaîne de personnes impliquées dans la production, par rapport aux partenaires de la situation de travail: témoins sans doute mais de quel procès et pour quelle finalité, acteurs éventuellement mais de quelle manière et dans quelles relations avec les autres agents de la situation?

Comolli ne cherchait pas à enfermer l'ensemble du cinéma dans une définition étroite de ses relations avec le travail : il mettait plutôt en question une certaine conception française du cinéma d'auteur exprimée à partir de la nouvelle vague et de la revue les Cahiers du cinéma. Cette conception fait du cinéma essentiellement un art et l'oppose ainsi à une reconnaissance du travail comme valeur positive. Le triomphe en France de

Images du travail, travail des images, 11 | 2021 
ce cinéma d'auteur correspond, comme le souligne justement le sociologue Yann Darré, à une transformation de la société et à un changement dans la nature des publics et de leurs choix. À la fin des années cinquante, il y a une baisse significative, de plus de la moitié, de la fréquentation des salles de cinéma. Selon Darré : «Cette baisse... est sélective: c'est d'abord le public populaire qui déserte les salles au profit de la télévision, tandis que le cinéma, légitimé comme art et pas seulement par les Cahiers $d u$ cinéma, devient la pratique culturelle par excellence de la bourgeoisie en ascension, parce qu'à la fois légitime et d'un accès relativement facile... Cette mutation du public permet l'existence du cinéma que prônent les jeunes-turcs, un cinéma où l'on parle de ce que l'on connaît et donc de son milieu, à un public qui peut s'y reconnaître » (2003, 124-125). Ce phénomène devrait être mis en relation, non seulement avec les prémices de la disparition de la classe ouvrière comme sujet principal de l'histoire mais également avec l'élargissement et la modélisation des comportements d'une classe intermédiaire, très largement urbanisée et l'amplification du secteur tertiaire. Ce sera le terrain de diffusion d'une culture moyenne internationale, ferment d'une mondialisation en cours mais encore masquée à cette époque.

On comprend bien ainsi que le travail, conçu comme activité aliénante de la personne soumise à la contrainte dominante du capital, celui auquel serait assujettie la classe ouvrière, n'est plus un sujet privilégié de la narration et les ouvriers eux-mêmes n'y sont plus que rarement représentés. Il faudrait nuancer néanmoins cette remarque en évoquant le film récent de Mathieu Gokalk, Rien de personnel (2009), mettant en scène la classe dirigeante au travail, des cadres victimisés par un formatage à outrance qui anéantit leur personne en sérialisant les individus anesthésiés par la compétition. Signe d'une évolution sinon même d'une véritable mutation des relations sociales et économiques dominées par la mondialisation.

Une exception notable cependant, celle du cinéma anglais dont la production, tout en diminuant considérablement, conserve une certaine importance à la description de la classe ouvrière et de la petite-bourgeoisie, notamment dans les films de Ken Loach. On peut y voir une persistance de la tradition sociale du cinéma britannique inaugurée dans les années trente par John Grierson et son équipe fameuse au sein de laquelle se trouvaient Alberto Cavalcanti et Robert Flaherty. Il est vrai que ces films montrent plutôt les conséquences dramatiques de la désindustrialisation britannique au cours de la seconde moitié du XXe siècle et qu'il s'agirait ainsi d'une démonstration a contrario de la valeur du travail par la mise en évidence des méfaits de sa destruction.

31 Nous voyons donc la difficulté à donner une identification générique à ce que devrait être le cinéma, échappant à la fois à ses lieux et conditions d'exercice et aux identités spécifiques de ses utilisateurs. Il s'agit d'un mode d'expression, d'exploration, de découverte dont les limites, les intentionnalités, les champs d'application et d'implications sont variables et renvoient nécessairement aux circonstances et aux situations de son usage.

On peut poser la question des différentes manières de traiter du travail au cinéma et tenter de les identifier dans un contexte particulier, il ne semble par contre pas très pertinent de s'interroger sur ce que serait la relation du cinéma en général avec le travail en général. Situer la démonstration, dans le temps et l'espace, permet de mieux identifier les pratiques, leur portée et leurs significations. Il ne s'agit donc pas du cinéma en tant que tel mais de l'usage spécifique d'un mode d'expression particulier 
auquel nous devons porter attention. Sans doute ce mode d'expression a-t-il des limites historiques et/ou sociologiques et ses conditions d'exercice sont-elles relatives.

Qualifiant les conséquences de ce que seraient les images pour Gilles Deleuze, Jacques Rancière écrit: "Les images donc sont proprement les choses du monde. Une conséquence doit s'en tirer logiquement : le cinéma n'est pas le nom d'un art, c'est le nom du monde » $(2001,46)$. En modifiant sensiblement cette conséquence, on pourrait dire qu'un film (et non le cinéma comme tel non plus que toute autre forme d'expression) est une proposition adéquate pour nommer un certain monde. Ainsi, pour la Nouvelle Vague, le cinéma n'était pas une représentation de la réalité mais une réalité. C'était la revendication de François Truffaut proclamant «le cinéma c'est la vie ». Le film est conçu comme une pensée en action, possibilité étonnante de surmonter la distinction dualiste occidentale entre la réflexion et sa mise en œuvre. Le film pourrait être l'expression visible et pratique d'une réunification de ces domaines d'abord séparés puis de plus en plus opposés, le travail et la vie.

Chaque film est un ensemble de propositions organisées différemment suivant l'endroit et le moment où on les reçoit. Paradigme interprétatif, il introduit dans l'ordonnancement momentané des choses du monde un élément de réalité supplémentaire qui en modifie, plus ou moins, mais nécessairement la constitution. Toute production imagétique - mais aussi toute forme de création - serait au-delà de la description et de l'analyse, comme une suggestion pour la transformation de l'espace vécu à l'intérieur duquel elle se déroule. Ce serait en définitive une intervention, un travail sur et dans le monde, une création constamment en cours, une réconciliation entre la vie et le travail dont les ouvriers de Chronique d'un été constataient amèrement l'impossibilité pour eux. En ce sens, un film ne cesse de vivre et de se transformer ou tout au moins de suggérer différentes compréhensions de ce qu'il propose. Il s'agit d'un corps constitué de propositions qui s'éclairent réciproquement et différemment suivant les lieux, les ordres, les instruments et les distances de rencontre et de lecture.

\section{Le cinéma comme entreprise}

Le cinéma, comme mode général d'expression, est capable de construire une situation dont l'autonomie se manifeste à partir d'une modification concrète de la matière en représentation. Sa capacité imagétique le constitue comme lieu de rencontre d'expériences multiples. Il est en effet un discours au même sens que celui de la parole ou de l'écriture mais il est également une mise en scène des choses du monde. Il opère sur la réalité des choses et des personnes et il est l'objet d'une production véritable qui le situe dans l'univers industriel et économique. Il possède aussi une capacité d'expérimentation sur un ordre extérieur dont il parle mais dont il est également le produit comme objet et comme re-présentation, sans pour autant se contenter de l'imiter ni tenter de l'absorber. Il propose et dispose des situations qui font effet sur la réflexion, les sentiments, provoquent des émotions, interrogent. Il implique des personnes et des personnages inventés ou réels qui ne sont pas sans relation avec les spectateurs et leurs propres relations quotidiennes, leurs préoccupations sociales, politiques, éthiques. Il construit et déconstruit une matérialité apparente dont il dit une certaine histoire, dont il propose en même temps la conservation, l'interprétation et la transformation. 
36 Dire une ville, un appartement, un campement de pêcheurs, en montrer les orientations, les ombres et les lumières, franchir les murs, ouvrir les portes, passer les limites, interroger ses habitants, les confronter entre eux, les provoquer ou les masquer, choisir des lieux plutôt que d'autres, nouer des intrigues ou même produire le mensonge d'une histoire comme aurait dit Jean Epstein (1974), signifier, faire percevoir, sentir, ressentir le tremblement des ombres, le rythme des respirations, les souffles des confidences ou les fracas des passions, interroger la durée, l'obscurité des actes et des sentiments, susciter directement l'attente, l'inquiétude ou l'espérance, le désir et la répulsion, faire entendre, attendre, espérer, interroger... Le cinéma n'est pas séparé comme le pensait Deleuze entre l'image-mouvement et l'image-temps, il est de façon indiscernable et simultanément les deux, même lorsque de façon plus ou moins délibérée le réalisateur ou le spectateur tentent de privilégier un aspect plutôt qu'un autre. La différence justement du cinéma d'avec d'autres langages serait de pouvoir proposer une réalité irrémédiablement syncrétique et plurielle, indéfinie, là où les autres langages tentent constamment une reconstruction ordonnée par un irrépressible désir d'homogénéité sélective réduisant la profusion et l'absence de signification du chaos universel à des logiques finalisées ou plutôt finalistes. Il est un travail en cours constamment ou plutôt il serait le cours d'un travail, celui d'un doute à la fois sur ses propres origines, ce dont il parle, ce qu'il dé-montre et ce vers quoi il tente de se diriger, c'est à dire nous, les spectateurs d'aujourd'hui ou de demain, interrogés par des virtualités imagétiques inquiétantes. Leur capacité extraordinaire est de se répéter sans cesse sans jamais signifier la même chose, sans jamais offrir ni parcourir les mêmes espaces.

37 Le cinéma permet de s'approcher de ce fourre-tout de l'existence sans nécessairement y trouver un sens mais en permettant au spectateur de s'interroger sur les manières de s'en accommoder et d'en explorer les non-sens. Il peut inventer s'il le faut un sens transitoire mais qui n'aura pas d'autre sens que celui de rassurer un instant sans convaincre jamais de sa nécessité et d'une pertinence qui en ferait une réalité « objective ». Peut-être pourrait-on se hasarder à dire que le cinéma en tant que tel serait la poursuite sans cesse recommencée, constamment inachevée d'une essence d'un monde à jamais inatteignable et toujours renvoyée à la pluralité innombrable de ses existences possibles. C'était une leçon de Sergueï Eisenstein qui incluait la participation du spectateur dans l'élaboration significative d'un film. Il exprimait déjà ce que je propose comme le nécessaire inachèvement de toute entreprise filmique concevable, conséquence autant que prémisse de l'inachèvement de toute réalité possible. Ce serait sans doute le chemin provisoirement spécifique d'un langage imagétique offrant simplement de nouveaux parcours et non pas de nouveaux objets à l'inquiétude des autres langages.

38 À l'encontre des autres langages, le langage cinématographique ne tente pas un transfert analytique du monde pour révéler ce qui en serait l'ordre régisseur. Bien au contraire, il propose la captation de ses données multiples, dans toutes leurs dimensions et même celles qui échapperaient à l'ordre concret de l'histoire. Il peut bien suggérer l'éclatement de la continuité apparente du temps ainsi que des diffractions narratives traversant les frontières des faits possibles et de l'imaginaire. Une définition en ce sens semblerait, à l'opposé de ce que je prétends, lui reconnaître une spécificité générique, il y aurait finalement ce cinéma en général dont on pourrait parler comme tel. À vrai dire, il s'agit seulement d'une disposition stratégique 
provisoire qui a fait du cinéma comme un signe avant-coureur de la mondialisation des communications et donc un instrument plus susceptible que d'autres d'explorer et de reproduire un large spectre des transformations en cours, notamment dans le domaine des perceptions et des conceptions de l'espace. Le travail de ce cinéma, constamment à l'œuvre et inventé par ceux qui le font et le voient, est d'aller au-delà du visible immédiat et de la reproduction virtuelle: sa mort peut être annoncée s'il devient prisonnier de ce qui existe déjà, s'il cesse de travailler, d'interroger et de contribuer à produire la réalité. Sans doute pourrait-il être avant tout entreprise, mais en tant que tel, rappelons qu'il peut tout aussi bien se consacrer au maintien de l'ordre qu'à sa mise en question.

\section{BIBLIOGRAPHIE}

Darré Y. (2003) «Le cinéma, l'art contre le travail, quand la société fait son cinéma », Mouvements, $\mathrm{n}^{\circ} 27 / 28,2003$, p. 124-125.

Epstein J. (1974) Écrits sur le cinéma, Seghers, 1974.

Piault M.-H. (2000) Anthropologie et cinéma. Passage à l'image, passage par l'image, Nathan, 2000.

Piault M.-H. (2004) « Un cinéma en travail ? Quelques réflexions sur l'entreprise imagétique à partir du film de Jean Rouch et Edgar Morin : Chronique d'un été », articles du comité du film ethnographique (en ligne), juillet 2004. [Reprint: “Um cinema em trabalho? Algumas reflexoes sobre o empreendimento imagético a partir do filme de Jean Rouch et Edgar Morin : Crônica de um verão", Rio, Cadernos de antropologia e Imagem, vol. 19, n 1, p. 25-46, 2004].

Rancière J. (2001) La fable cinématographique, Seuil, 2001.

Rouch J. \& Morin E. (1960) Chronique d'un été, Paris, Argos films, 16 mm, $90 \mathrm{~min}$.

Rouch J. \& Morin E. (1962) « Le cinéma de l'avenir? » in Chronique d'un été de Jean Rouch et Edgar Morin, Domaine Cinéma 1, Inter Spectacles, p. 44-52.

Rouch J., France C. (de) (1979) Pour une anthropologie visuelle, Mouton, p. 53-71.

\section{NOTES}

1. Baroncelli (de) J., Le Monde, 27/09/61. D’abord réalisateur reconnu des années 1930-1940, il fut l'un des initiateurs en France d'une réflexion générale sur le cinéma.

2. Il s'agit d'ailleurs de l'Amérique dans son ensemble puisque, par exemple, les éditeurs français publient Jorge Amado, Mario de Andrade, Borges, Coccioli, Gilberto Freyre, Néruda, que les galeries exposent Orozco et Ribeira peu de temps avant la venue d'un "nouveau cinéma ", argentin, brésilien, chilien, mexicain...

3. Cf. pour plus de détails Rouch \& Morin (1962). On y trouvera les dialogues complets du film et de quelques scènes coupées au montage, des commentaires des réalisateurs et des acteurs ainsi que certaines critiques qui ont suivi la sortie publique du film. 
4. Lors de la projection de Chronique d'un été que j'avais organisée à la Cinémathèque française à Paris (mars 2004), en hommage à Jean Rouch qui venait de disparaittre, Marceline a confirmé sa mise en scène dont l'intensité émotionnelle pour le spectateur ne venait pas d'une spontanéité supposée mais de l'intensité et de la réalité terrible du récit lui-même, de son contenu et de son interprétation. Edgar Morin déclarait alors avec émotion : " Aujourd'hui je dois dire que ce film est le film de Rouch... » On perçoit bien en effet Chronique d'un été comme une œuvre essentiellement rouchienne.

5. Michel Brault, cinéaste canadien dont le film Les Raquetteurs (1958) avait conduit Edgar Morin vers la notion de " cinéma vérité ». Il avait rejoint l'équipe de réalisation en apportant des " micros-cravates » tout récents. Avec la caméra légère et relativement insonorisée de l'ingénieur Coutant, cela permit les premières expérimentations de tournage léger en extérieur avec son synchrone. Brault, Rouch et l'ingénieur du son Michel Fano rencontraient pendant le tournage le constructeur suisse Stéphane Kudelski qui, stimulé par leur enthousiasme, mettait au point un an plus tard un magnétophone professionnel portable et synchrone, le fameux Nagra qui allait libérer techniquement ce nouveau cinéma.

6. Toutes les citations sont empruntées à Rouch, Morin, 1962.

\section{RÉSUMÉS}

En analysant le célèbre film de Jean Rouch et d'Edgar Morin, on peut y voir l'une des premières expressions d'un malaise qui commence à saisir une partie de la jeunesse et de la classe ouvrière. À l'aube des années 1960, la France connaît en effet des transformations radicales marquées par la décolonisation, l'exode rural massif et la reconstruction de ses villes et de ses industries. La jeunesse tourne le dos à la guerre et découvre le plaisir, le voyage et les tentations du consumérisme mais aussi les premiers doutes relatifs au progrès et à l'organisation taylorienne du travail. Grâce au dispositif du film visant à donner la parole et grâce aux innovations technologiques du cinéma direct, Chronique d'un été saisit les premiers signes des mutations en cours qui s'affirmeront notamment en 1968. Les deux chercheurs/réalisateurs y voient la possibilité d'une prise de conscience, celle de rencontrer l'autre sans l'assimiler ou le dominer.

By analysing Jean Rouch and Edgar Morin's famous film, we can see one of the first expressions of awkwardness that is beginning to affect part of the youth and the working class. At the dawn of the 1960s, France was undergoing radical transformations marked by decolonisation, a massive rural exodus and the reconstruction of its cities and industries. Young people turned their back on the war and discovered pleasure, travel and the temptations of consumerism, but also the first doubts regarding progress and the Taylorian organisation of work. Thanks to its device that aims to give voice to them and thanks to the technological innovations of direct cinema, Chronique d'un été captures the signs of the ongoing mutations, which were asserted in particular in 1968. The two researchers/directors see in it a possible awareness, that of meeting the other without assimilating or dominating him. 
INDEX

Mots-clés : travail, cinéma direct, après-guerre, décolonisation, jeunesse, altérité

Keywords : work, direct cinema, post-war, decolonisation, youth, otherness

\section{AUTEUR}

\section{MARC-HENRI PIAULT}

Décédé le 4 novembre 2020, Marc-Henri Piault s'est beaucoup intéressé aux images du travail comme anthropologue, cinéaste et directeur de recherche au CNRS. Ses terrains l'ont mené à travers le monde, de l'Afrique occidentale (migrations, formations politiques précoloniales, cultes de possession) à la France (identité régionale et représentation culturelle) et au Brésil (croyances, appartenances, identités et conflits). Il a présidé le Comité du film ethnographique au sein duquel il transforme le nom du festival en l'honneur de son créateur Jean Rouch et afin de l'ouvrir plus largement aux questions de cinéma. Il fut responsable de l'Association Française des Anthropologues, du Journal des Africanistes, du Journal des Anthropologues et l'auteur d'une centaine de publications en français, anglais, portugais, espagnol et italien. 